\title{
Consistent Histories and Contrary Inferences
}

\author{
G. Nisticò \\ Istituto Nazionale Fisica Nucleare, Italy \\ Dipartimento di Matematica, Università della Calabria, via P. Bucci 30b, 87036, Rende (Italy)
}

Received on 26 January, 2005

\begin{abstract}
To perform a more transparent analysis of the problems raised by contrary inferences within Consistent History approach to Quantum Theory, we extend the formalism of the conceptual basis. According to our analysis, the conceptual difficulties arising from contrary inferences are ruled out.
\end{abstract}

\section{Introduction}

Consistent History Approach to quantum theory, introduced in explicit form by Griffiths [1], provides an extension of the interpretation of the formalism of quantum mechanics. While standard quantum theory is based on the concept of event, represented by a projection operator $E$ of the Hilbert space $\mathcal{H}$ describing the system, $\mathrm{CHA}$ is based on the concept of history, which is a finite ordered sequence $h=\left(E_{1}, E_{2}, \ldots, E_{n}\right)$ of events. Suitable families of histories can be selected by means of a criterion of consistency. According to CHA, only the histories of a consistent family have physical meaning. The occurrences of histories are the empirical facts the theory is concerned with.

One of the aims of CHA is to solve some conceptual difficulties of standard quantum mechanics. Griffiths and Omnès [2] argue that the famous quantum measurement problem [3] is solved by the extended interpretation provided by CHA.

However, the new theory raised some criticisms. One of the most debated problems is linked to the so called contrary inferences, illustrated in section 3 below. The debate provoked by this problem did not reach a shared conclusion. We think that a better clarity can be obtained if some natural concepts of CHA are better formalized, so that to allow a more transparent analysis of the debated questions.

We do this by extending the formalism of the conceptual basis of CHA with the introduction of the notion of support. Starting from it, new tools can be developed allowing a deeper analysis of some questions. Also the problem of contrary inferences can be submitted to such an analysis. Our result is that contrary inferences do not entail conceptual difficulties.

The plan of the paper is the following. In section 2 the basic concepts of CHA are outlined. The problem of contrary inferences is described is section 3. The notion of support of a family of histories is introduced in section 4 , where the formalism stemming from this notion is developed. In section 4.2 some general implications of the extended formalism are derived. A deeper undestanding of CHA notion of compatible families is obtained in section 4.3.

Finally, the problem of contrary inferences is analyzed in section 5 on the basis of the extended formalism, showing that the related difficulties are ruled out.

\section{Basic concepts of CHA}

Let $\mathcal{H}$ be the Hilbert space which describes the physical system according to standard quantum theory. Throughout this paper we assume that $\mathcal{H}$ has a finite dimension $N$; moreover, the Heisenberg picture is adopted. Fixed a finite sequence of times $t_{1}, t_{2}, \ldots, t_{n}$, let us consider for each time $t_{k}$ a decomposition of the identity $\mathbf{E}_{k}=\left\{E_{k}^{(1)}, E_{k}^{(2)}, \ldots, E_{k}^{\left(i_{k}\right)}\right\}$ which is a finite set of projection operators of Hilbert space $\mathcal{H}$, with $E_{k}^{(i)} \perp E_{k}^{(j)}$ if $i \neq j$ and $\sum_{i=1}^{i_{k}} E_{k}^{(i)}=1$. A family $\mathcal{C}$ of histories, generated by $\mathbf{E}_{1}, \mathbf{E}_{2}, \ldots, \mathbf{E}_{n}$, is the set of all sequences $h=\left(E_{1}, E_{2}, \ldots, E_{n}\right)$ such that $E_{k}=\sum_{\text {some }_{i}} E_{k}^{(i)}$. Projection operator $E_{k}$ in a history $h$ represents an event susceptible of occurring at time $t_{k}$. When every event $E_{k}$ constituting a history $h$ is just an event of $\mathbf{E}_{k}$, i.e. if $E_{k} \in \mathbf{E}_{k}$ for all $k=$ $1,2, \ldots, n$, then $h$ is called elementary history. Hence the set $\mathcal{E}$ of all elementary histories of $\mathcal{C}$ is the cartesian product $\mathcal{E}=$ $\mathbf{E}_{1} \times \mathbf{E}_{2} \times \cdots \times \mathbf{E}_{n}$. For every history $h=\left(E_{1}, E_{2}, \ldots, E_{n}\right)$, by $\mathbf{h}$ we denote the following subset of elementary histories: $\mathbf{h}=\left\{\hat{h}=\left(\hat{E}_{1}, \hat{E}_{2}, \ldots, \hat{E}_{n}\right) \in \mathcal{E} \mid \hat{E}_{k} \leq E_{k}\right\}$. Two histories $h_{1}=\left(E_{1}, E_{2}, \ldots, E_{n}\right), h_{2}=\left(F_{1}, F_{2}, \ldots, F_{n}\right) \in \mathcal{C}$ are summable if they differ in only one place, say $k$, hence $E_{j}=F_{j}$ for all $j \neq k$, and $E_{k} \perp F_{k}$; in such a case their sum is $h_{1}+h_{2}=\left(E_{1}, E_{2}, \ldots, E_{k}+F_{k}, \ldots, E_{n}\right) \in \mathcal{C}$. The histories $h_{1}$ and $h_{2}$ are said to be alternative if there is $k$ such that $E_{k} \perp F_{k}$.

Let $h=\left(E_{1}, E_{2}, \ldots, E_{n}\right)$ be a commutative history, i.e. such that all $E_{k}$ commute with each other. According to quantum theory, the statement " $h$ occurs" means that all events $E_{1}, E_{2}, \ldots, E_{n}$ occur. Therefore, $h$ is identified with the single event $E_{1} \cdot E_{2} \cdots E_{n}=E_{1} \wedge E_{2} \wedge \cdots \wedge E_{n}$. Though the mathematical notions of $\mathrm{CHA}$ are given within the usual quantum theoretical formalism, standard quantum theory is unable to consider and describe the occurrence of a history when it is not commutative. However, in the case that $\mathcal{C}$ is weakly decohering, i.e. if $\operatorname{Re}\left(\operatorname{Tr}\left(C_{h_{1}} C_{h_{2}}^{*}\right)\right)=0$ for all $h_{1}, h_{2} \in \mathcal{E}, h_{1} \neq h_{2}$, the functional $p_{\mathcal{C}}: \mathcal{C} \rightarrow[0,1]$, $p_{\mathcal{C}}(h)=\frac{1}{N} \operatorname{Tr}\left(C_{h} C_{h}^{*}\right)$ behaves as a probability which ex- 
tends the probability predicted by standard quantum theory for commutative histories [4]. The basic principle of CHA establishes that when family $\mathcal{C}$ satisfies the mathematical criterion of being weakly decohering then it is consistent, i.e.

(I) the set of all elementary histories of $\mathcal{C}$ is a "sample space of mutually exclusive elementary events, one and only one of which occurs" [8].

The occurrence of history $h$ means that an elementary history $\hat{h} \in \mathbf{h}$ occurs. Here the physical meaning of the notion of occurrence of a history, involved in (I), is the following

(O) A given history $h=\left(E_{1}, E_{2}, \ldots, E_{n}\right)$ occurs if all events $E_{1}, E_{2}, \ldots, E_{n}$ objectively occur at respective times $t_{1}, t_{2}, \ldots, t_{n}$. The occurrence of a history is an objective physical fact, independent of the performance of a measurement which reveals this occurrence.

Accordingly, if $\mathcal{C}$ is consistent, any occurrence (or nonoccurrence) of an elementary history in $\mathcal{C}$ must be referred to an individual, concrete sample of the physical system.

Remark 1. The probability of occurrence of a history $h$, $p_{\mathcal{C}}(h)=\frac{1}{N} \operatorname{Tr}\left(C_{h} C_{h}^{*}\right)$, is clearly independent of the consistent family it belongs to. However, the symbol $\mathcal{C}$ is kept in the formula for future convenience, as explained in remark 2 below.

\section{Contrary inferences}

To make predictions about occurrences of histories, the initial data, i.e. the known information about the physical system, are expressed as sentences involving histories of a consistent family $\mathcal{C}$, and assumed as true, i.e. objectively realized sentences. Conclusions can be derived by means of logical reasonings in which histories of $\mathcal{C}$ are regarded as events of a classical sample space and by interpreting the number $p_{\mathcal{C}}(h)=\frac{1}{N} \operatorname{Tr}\left(C_{h} C_{h}^{*}\right)$ as the probability of occurrence of history $h$. These conclusions are the theoretical predictions to be regarded as objectively realized sentences.

However, the histories needed to express the same initial data belong, in general, also to another consistent family $\mathcal{C}^{\prime}$. Therefore, another set of conclusions can be obtained by using $\mathcal{C}^{\prime}$ instead of $\mathcal{C}$. A conclusion of this new set could contradict some conclusion derived from $\mathcal{C}$. To avoid these conflicts, $\mathrm{CHA}$ introduces the following rule.

Single Family Rule. All valid physical inferences are those obtained by using a single consistent family $\mathcal{C}$. In general, different conclusions drawn by using distinct consistent families do not hold together.

The possibility of contrary inferences is the main criticism opposed to CHA. They are a particularly effective example of the conflicts described above. Let us briefly describe them. Suppose that $\mathcal{C}_{1}$ and $\mathcal{C}_{2}$ are two different weakly decohering families such that $h_{1}=\left(E_{0}, E_{1}, E_{2}\right) \in \mathcal{C}_{1}$ and $h_{2}=\left(E_{0}, F_{1}, E_{2}\right) \in \mathcal{C}_{2}$, with $E_{1} \perp F_{1}$, and $h_{0}=$ $\left(E_{0}, 1, E_{2}\right) \in \mathcal{C}_{1} \cap \mathcal{C}_{2}$. A. Kent [5] was able to find examples in which the conditional probabilities $p_{\mathcal{C}_{1}}\left(h_{1} \mid h_{0}\right)=\frac{p_{\mathcal{C}_{1}}\left(h_{1}\right)}{p_{\mathcal{C}_{1}}\left(h_{0}\right)}$ and $p_{\mathcal{C}_{2}}\left(h_{2} \mid h_{0}\right)=\frac{p_{\mathcal{C}_{2}}\left(h_{2}\right)}{p_{\mathcal{C}_{2}}\left(h_{0}\right)}$ are both 1 . Therefore, according to CHA we may state that if $h_{0}$ occurs, then also $E_{1}$ occurs within the family $\mathcal{C}_{1}$, but then also $F_{1}$ occurs within the family $\mathcal{C}_{2}$; on the other hand, $E_{1} \perp F_{1}$ means that the occurrence of $E_{1}$ excludes the occurrence of $F_{1}$ : then we have two inferences which are contrary to each other. Contrary inferences do not entail logical incoherence for CHA because they take place in different consistent families, and thus the contradiction arises only by violating the single family rule.

The presence of contrary inferences in CHA is at the root of a rather lively debate. According to Kent, their occurrence makes it "hard to take it [CHA] seriously as a fundamental theory in its present form" [5]. And about single family rule, which formally prevents contrary inferences from yielding theoretical contradiction [6], "any formalism [...] can be made free from contradiction by such a restriction" [7]. The replies of Griffiths are essentially based on the following arguments.

1. The derivation of the contradiction violates the single family rule. In particular, the problem arises because Kent assigns to 'contrary' histories $h_{1}$ and $h_{2}$ the classical-logic meaning of word contrary, i.e. that the occurrence of $h_{1}$ always implies the non-occurrence of $h_{2}$. But this cannot be done because $h_{1}$ and $h_{2}$ cannot be compared without violating single family rule 3 .

2. "The conceptual difficulty goes away if one supposes that the two incompatible frameworks are being used to describe two distinct physical systems that are described by the same initial data" [9].

The critics of CHA were not satisfied by these arguments. To synthetically explain the reason for such a disagreement, let us consider the example of contrary inferences above outlined and two physicists, Alice and Bob. Suppose that the known data about a single physical system $s$ are that $E_{0}$ and $E_{2}$ occur, i.e. $h_{0}$ occurs. In order to establish whether $E_{1}$ occurs or not, Alice uses family $\mathcal{C}_{1}$ and, in accordance with $\mathrm{CHA}$, she finds that $E_{1}$ occurs. Bob, for the same individual system $s$, chooses $\mathcal{C}_{2}$, and he concludes that $F_{1}$ occurs. The fact that $E_{1}$ occurs or $F_{1}$ occurs seems to depend on the physicist. But, according to $\mathrm{CHA}$ itself, the occurrence of a history, once established by means of the theory, is to be considered an objective fact. As a consequence, both $E_{1}$ and $F_{1}$ should occur. But everybody rejects this conclusion because $E_{1} \perp F_{1}$.

\section{Extended formalism}

Now we introduce the notion of support of a family of histories. In such a way we give formal content to some concepts, naturally stemming from the basic principles of $\mathrm{CHA}$ reflected by (I) and (O), which are not formalized in the standard formulation of CHA. Two basic axioms (1 and 2) shall be formulated as statements which arise from the physical meaning of support. Such an extension of the formalism supplies us with new tools which make possible a conceptually transparent analysis of some questions in CHA. According to 
the results of this analysis, the conceptual difficulties linked to contrary inferences are ruled out.

\subsection{The notion of support}

Given a family $\mathcal{C}$ and a concrete specimen $s$ of the physical system, there are two mutually exclusive possibilities: for this particular $s$ either

i) one elementary history $h$ of $\mathcal{C}$ occurs and all other elementary histories do not occur,

or

ii) no one of the elementary histories of $\mathcal{C}$ occurs.

In case (i) we say that the histories of $\mathcal{C}$ make sense for this $s$; in case (ii) we say that the histories of $\mathcal{C}$ do not make sense.

For instance, if the physical system is a silver atom entering a Stern and Gerlach apparatus, we may consider two families of histories.

Family $\mathcal{C}^{(a)}$, which is the family generated by the following elementary histories

$h_{1}^{(a)}=$ "the atom emerges from the apparatus with spin up"

$h_{2}^{(a)}=$ "the atom emerges from the apparatus with spin down".

Family $\mathcal{C}^{(b)}$, which is the family generated by the following elementary histories

$h_{1}^{(b)}=$ "the atom is democratic"

$h_{2}^{(b)}=$ "the atom is republican".

For this particular $s$ family $\mathcal{C}^{(a)}$ makes sense. On the contrary, family $\mathcal{C}^{(b)}$ does not.

By support of $\mathcal{C}$ we mean the set $b(\mathcal{C})$ of all concrete specimens of the physical system for which $\mathcal{C}$ makes sense. The introduction of set $b(\mathcal{C})$ allows us to formally express the consistency of a family by means of a simple definition which reflects the meaning of consistency expressed by (I) and (O).

Definition 1. A family $\mathcal{C}$ is consistent if and only if $b(\mathcal{C}) \neq \emptyset$.

As an evident insight, the (sufficient) condition which makes the conclusions of logical reasonings based on a family $\mathcal{C}$ valid for a specimen $s$ of the physical system is

$$
s \in b(\mathcal{C}) .
$$

Remark 2. As a consequence, also the number $p_{\mathcal{C}}(h)=$ $\frac{1}{N} \operatorname{Tr}\left(C_{h} C_{h}^{*}\right)$ can be interpreted as probability of occurrence of $h$ only if $s \in b(\mathcal{C})$. For this reason it makes sense to keep symbol $\mathcal{C}$ in this formula.

Now we deduce a natural implication for the supports of two families $\mathcal{C}_{1}$ and $\mathcal{C}_{2}$ such that $\mathcal{C}_{1} \subseteq \mathcal{C}_{2}$. We consider the case in which $b\left(\mathcal{C}_{2}\right) \neq \emptyset$. For every $s \in b\left(\mathcal{C}_{2}\right)$, there is only one elementary history $h_{2}(s)$ of $\mathcal{C}_{2}$ which occurs, and all other elementary histories of $\mathcal{C}_{2}$ do not occur. From $\mathcal{C}_{1} \subseteq \mathcal{C}_{2}$ it follows that all elementary histories of $\mathcal{C}_{1}$ form a set, denoted by
$\mathcal{E}_{1}$, of albeit non-elementary histories of $\mathcal{C}_{2}$. Only one history $h_{1} \in \mathcal{E}_{1}$ occurs, because there is a unique $h_{1} \in \mathcal{E}_{1}$ such that $h_{2}(s) \in \mathbf{h}_{1}$. Analogously we find that all other $h \in \mathcal{E}_{1}$ do not occur. Therefore, it is possible to state that only one elementary history of $\mathcal{C}_{1}$ occurs and all other $h \in \mathcal{E}_{1}$ do not occur for this individual system $s$; thus, by definition $1, s \in b\left(\mathcal{C}_{1}\right)$. We state the result of this argument as the following axiom.

Axiom 1. Let $\mathcal{C}_{1}, \mathcal{C}_{2}$ be two families of histories. Then

$$
\mathcal{C}_{1} \subseteq \mathcal{C}_{2} \quad \text { implies } \quad b\left(\mathcal{C}_{2}\right) \subseteq b\left(\mathcal{C}_{1}\right)
$$

Now we proceed to state another axiom. If $h \in \mathcal{C}$, by $b_{1}(h ; \mathcal{C})$ (resp., $\left.b_{0}(h ; \mathcal{C})\right)$ we denote the subset of $b(\mathcal{C})$ whose elements are the specimens for which $h$ occurs (resp., does not occur). It is obvious to assume that

$$
b_{0}(h ; \mathcal{C})=b(\mathcal{C}) \backslash b_{1}(h ; \mathcal{C}) \text { and } b(\mathcal{C})=\cup_{h \in \mathcal{E}} b_{1}(h ; \mathcal{C})
$$

According to $(\mathrm{O})$, the occurrence of $h=\left(E_{1}, E_{2}, \ldots, E_{n}\right)$ is equivalent to the occurrences of all events $E_{1}, E_{2}, \ldots E_{n}$ at the respective times $t_{1}, t_{2}, \ldots, t_{n}$, and then it is independent of the family which $h$ belongs to. Therefore, we can state the following axiom.

Axiom 2. If $h \in \mathcal{C} \cap \mathcal{C}^{\prime}$, then $b_{1}(h ; \mathcal{C}) \cap b_{0}\left(h ; \mathcal{C}^{\prime}\right)=$ $b_{1}\left(h ; \mathcal{C}^{\prime}\right) \cap b_{0}(h ; \mathcal{C})=\emptyset$.

Axiom 2 establishes that a history $h$ cannot occur as history of $\mathcal{C}$ and do not occur as history of $\mathcal{C}^{\prime}$, for the same specimen $s$.

\subsection{General implications}

Let $X$ be any set of histories. The family generated by $X$ is $\mathcal{C}(X)=\cap_{\{\mathcal{C} \mid X \subseteq \mathcal{C}\}} \mathcal{C}$. By axiom 1 we have that $h \in \mathcal{C}$ implies $b(\mathcal{C}) \subseteq b(\mathcal{C}(\{h\}))=\cup_{h \in \mathcal{C}}(\mathcal{C})$. Therefore, $b(h) \equiv$ $b(\mathcal{C}(\{h\}))$ is the set of all specimens of the physical system for which single history $h$ occurs or does not occur ( $h$ makes sense). By $b_{1}(h)$ (resp., $b_{0}(h)$ )we denote the subset of those systems for which $h$ occurs (resp., does not occur). Of course

$$
b_{0}(h)=b(h) \backslash b_{1}(h), \quad b_{1}(h)=b(h) \backslash b_{0}(h) .
$$

According to the present approach, the fact that a given history $h_{0}$ occurs (or does not occur) for a physical system $s_{0}$ means that a family $\mathcal{C}_{0}$ exists such that

$$
h_{0} \in \mathcal{C}_{0} \quad \text { and } \quad s_{0} \in b\left(\mathcal{C}_{0}\right) .
$$

Family $\mathcal{C}_{0}=\mathcal{C}\left(\left\{h_{0}\right\}\right)$ fullfils these requirements. All histories of $\mathcal{C}_{0}$ make sense for $s_{0}$. However, the eventuality that for a given system $s$ history $h \in \mathcal{C}$ occurs but $s \notin b(\mathcal{C})$ is logically possible. Therefore, the fact that a family $\mathcal{C}$ is consistent, and $h \in \mathcal{C}$ does not imply that the inferences obtained by means of reasonings based on $\mathcal{C}$ hold for an arbitrary physical system $s$ for which $h$ occurs.

Suppose that the following statement holds for $s$ : "history $h_{0}$ occurs" (initial data). Then $s \in b_{1}\left(h_{0}\right)$, and hence 
$s \in b\left(\mathcal{C}\left(\left\{h_{0}\right\}\right)\right)$, are true statements. Thus, all sentences obtained by logical deductions based on $\mathcal{C}\left(\left\{h_{0}\right\}\right)$ hold for $s$.

Family $\mathcal{C}\left(\left\{h_{0}\right\}\right)$ admits several refinements, i.e. various families $\mathcal{C}$ may exist such that $\mathcal{C}\left(\left\{h_{0}\right\}\right) \subseteq \mathcal{C}$. Deductions based on a refinement $\mathcal{C}$ in general do not hold for $s \in c\left(\mathcal{C}\left(\left\{h_{0}\right\}\right)\right)$; indeed, as already argued, $\mathcal{C}\left(\left\{h_{0}\right\}\right) \subseteq \mathcal{C}$ implies, by Axiom $1, b(\mathcal{C}) \subseteq b\left(\mathcal{C}\left(\left\{h_{0}\right\}\right)\right)$, and therefore $s \in b(\mathcal{C})$ does not necessarily follow. Since $\mathcal{C}\left(\left\{h_{0}\right\}\right) \subseteq \mathcal{C}$, all inferences obtained by reasoning with $\mathcal{C}\left(\left\{h_{0}\right\}\right)$ can be obtained with $\mathcal{C}$; this implies that the two set of sentences cannot contradict with each other; but inferences involving histories in $\mathcal{C} \backslash \mathcal{C}\left(\left\{h_{0}\right\}\right)$ in general do not make sense if $s \in b\left(\mathcal{C}\left(\left\{h_{0}\right\}\right)\right) \backslash b(\mathcal{C})$.

Here is a profound difference between the "coarsest" family $\mathcal{C}(X)$ compatible with the available initial data relative to a physical system $s$ and any refinement $\mathcal{C}$ of $\mathcal{C}(X)$ : conclusions drawn by using $\mathcal{C}(X)$ are true, i.e. objective facts, whereas the truth of conclusions obtained from a refinement is not ensured by the truth of the data $(X)$ alone. As a consequence, in the present approach Griffiths' prescription: "Use the smallest, or coarsest framework which contains both the initial data and the additional properties of interest in order to analyse the problem." [9] is not always valid.

\subsection{Compatibility of families}

Now we come to analyze the notion of compatibility of families.

Definition 2. Two consistent families $\mathcal{C}_{1}$ and $\mathcal{C}_{2}$ are compatible if a third consistent family $\mathcal{C}$ exists such that $\mathcal{C}_{1} \cup \mathcal{C}_{2} \subseteq \mathcal{C}$.

According to standard CHA, the conclusions drawn in different families hold together in the case that these families are compatible. By axiom 1, compatibility implies $b(\mathcal{C}) \subseteq$ $b\left(\mathcal{C}_{1}\right) \cap b\left(\mathcal{C}_{2}\right)$; therefore, the conclusions drawn from $\mathcal{C}_{1}$ and $\mathcal{C}_{2}$ certainly hold together for the systems $s \in b(\mathcal{C})$. However, if $s \in b\left(\mathcal{C}_{1}\right) \cap b\left(\mathcal{C}_{2}\right)$, then the predictions drawn in $\mathcal{C}_{1}$ and $\mathcal{C}_{2}$ hold together for this system $s$, no matter whether $\mathcal{C}_{1}$ and $\mathcal{C}_{2}$ are compatible or not. It is true that single family rule of CHA guarantees from conflicting inferences, but it makes the theory unable to describe this possibility.

Moreover, if $s \in b\left(\mathcal{C}_{1}\right)$ and $s \notin b\left(\mathcal{C}_{2}\right)$, then a conclusion drawn from $\mathcal{C}_{2}$ does not necessarily hold for this $s$.

\section{Analysis of contrary inferences}

Contrary inferences were discovered by Kent within the framework of standard CHA. To can discuss them on the basis of our extended formalism, we make coincide the concept of consistency of definition 1 with that of standard CHA. This identification is formally realized by means of the following axiom.

Axiom CHA. A family $\mathcal{C}$ is consistent, in the sense of definition 1 , if and only if it is weakly decohering. In this case $p_{\mathcal{C}}(h)=\frac{1}{N} \operatorname{Tr}\left(C_{h} C_{h}^{*}\right)$ is the probability of occurrence of history $h$.

We begin our analysis of contrary inferences by formally showing, within our approach, that two mutually orthogonal projections represent events which cannot occur simultaneously.

Let $E$ and $F$ be two mutually orthogonal projections. The family generated by $E$ and $F$, i.e. $\mathcal{C}(\{E, F\})$, has 3 elementary (one-event) histories: $\mathcal{E}=\{E, F, G=\mathbf{1}-(E+F)\}$; it is the smallest family containing $E$ and $F$. Then, following the argument of section 4.2, whenever both $E$ and $F$ make sense, all histories in $\mathcal{C}(\{E, F\})$ must make sense too. Therefore, the following proposition holds.

Proposition 1. If $E \perp F$, then $s \in b(E) \cap b(F)$ implies $s \in b(\mathcal{C}(\{E, F\}))$ and, therefore, $b_{1}(E) \cap b_{1}(F)=\emptyset$.

If families $\mathcal{C}_{1}$ and $\mathcal{C}_{2}$ in Kent's example of contrary inferences were compatible families, then we would have a contradiction.

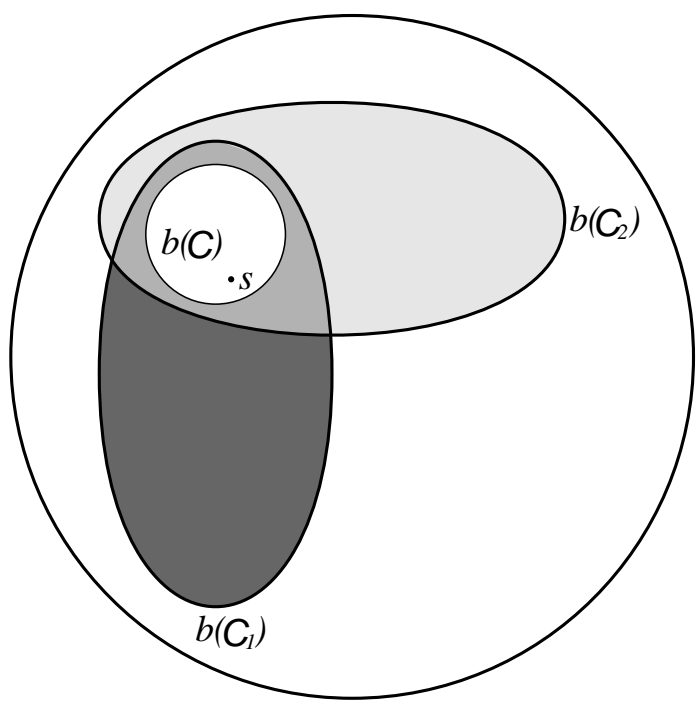

Figure 1. Compatible families.

Indeed, in this case a consistent family $\mathcal{C}$ would exist such that $\mathcal{C}_{1} \cup \mathcal{C}_{2} \subseteq \mathcal{C}$. By axiom 1 we get

$$
b(\mathcal{C}) \subseteq b\left(\mathcal{C}_{1}\right) \cap b\left(\mathcal{C}_{2}\right)
$$

Furthermore $h_{0}=\left(E_{0}, \mathbf{1}, E_{2}\right) \in \mathcal{C}$, because $h_{0} \in \mathcal{C}_{1} \cap \mathcal{C}_{2}$. Since $p_{\mathcal{C}}\left(h_{0}\right) \neq 0$, there would exist a specimen $\hat{s} \in$ $b_{1}\left(h_{0}\right) \cap b(\mathcal{C})$, and hence $\hat{s} \in b\left(\mathcal{C}_{1}\right) \cap b\left(\mathcal{C}_{2}\right)$ by (5). Therefore, we should conclude $\hat{s} \in b_{1}\left(E_{1}\right)$ and $\hat{s} \in b_{1}\left(F_{1}\right)$, because $p_{\mathcal{C}_{1}}\left(h_{1} \mid h_{0}\right)=p_{\mathcal{C}_{2}}\left(h_{2} \mid h_{0}\right)=1$. Thus we would have a contradiction with $b_{1}\left(E_{1}\right) \cap b_{1}\left(F_{1}\right)=\emptyset$ which follows from proposition 1 , since $E_{1} \perp F_{1}$. 


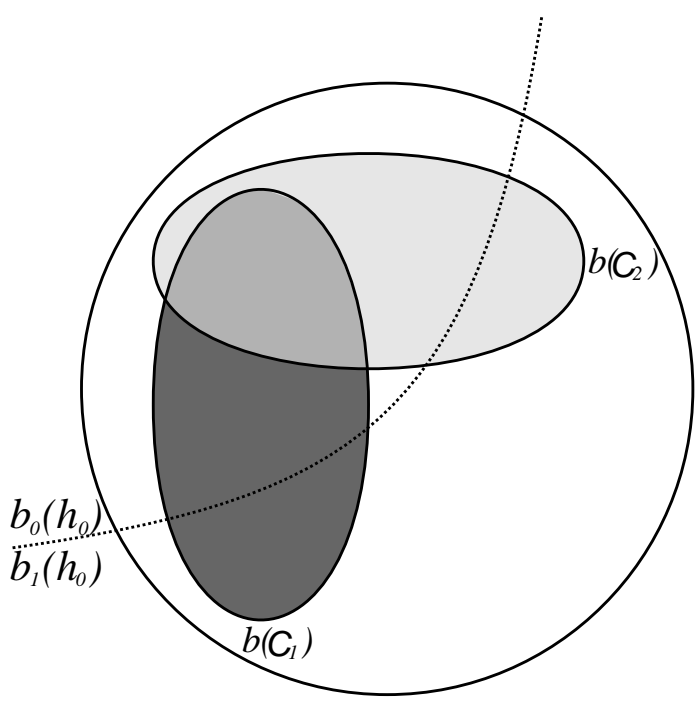

Figure 2. Non compatible families.

However, $\mathcal{C}_{1}$ and $\mathcal{C}_{2}$ in contrary inferences example are not compatible families, therefore previous argument does not apply. We can show that the occurrence of history $h_{0}=$ $\left(E_{0}, \mathbf{1}, E_{2}\right)$ does not give rise to conceptual difficulties. The foregoing argument shows that the condition to be satisfied to avoid the contradiction is

$$
b_{1}\left(h_{0}\right) \cap b\left(\mathcal{C}_{1}\right) \cap b\left(\mathcal{C}_{2}\right)=\emptyset .
$$

If $\mathcal{C}_{1}$ and $\mathcal{C}_{2}$ are not compatible, (6) is logically consistent with the occurrence of $h_{0}$. Indeed, from

$$
h_{0} \in \mathcal{C}_{1} \cap \mathcal{C}_{2}
$$

by axiom 1 we are simply led to

$$
b\left(\mathcal{C}_{1}\right) \cup b\left(\mathcal{C}_{2}\right) \subseteq b\left(h_{0}\right) .
$$

This relation is consistent with (6), contrary to what happen if $\mathcal{C}_{1}$ and $\mathcal{C}_{2}$ were compatible. Indeed, when $h_{0}$ occurs, i.e. if $s \in b_{1}\left(h_{0}\right) \subseteq b\left(h_{0}\right)$, we have 3 mutually exclusive and exhaustive possibilities: p) $\quad s \in b\left(\mathcal{C}_{1}\right)$. In this case $p_{\mathcal{C}_{1}}\left(h_{1} \mid h_{0}\right)=1$ must hold (see remark 2) and this entails that $E_{1}$ occurs. The consistency with (6) merely requires that $s \notin b\left(\mathcal{C}_{2}\right)$ so that $p_{\mathcal{C}_{2}}\left(h_{2} \mid h_{0}\right)=1$ (' $h_{0}$ occurs implies $F_{1}$ occurs'), does not hold for this $s$. Regards to the occurrence of $F_{1}$ there are two possibilities:

$\left.\mathrm{p}_{1}\right) s \in b\left(F_{1}\right) \backslash b\left(\mathcal{C}_{2}\right)$. This possibility is consistent because $F_{1} \in \mathcal{C}_{2}$ implies $b\left(\mathcal{C}_{2}\right) \subseteq b\left(F_{1}\right)$. In this case axiom 2 and proposition 1 imply that $F_{1}$ does not occur, i.e. $s \in b_{0}\left(F_{1}\right)$;

$\left.\mathrm{p}_{2}\right) s \notin b\left(F_{1}\right)$, therefore $F_{1}$ does not make sense, i.e. it neither occurs nor does not occur.

q) $s \in b\left(\mathcal{C}_{2}\right)$. In this case $p_{\mathcal{C}_{2}}\left(h_{2} \mid h_{0}\right)=1$ holds, and then $F_{1}$ occurs. We have for $E_{1}$ the same conclusions of item (p) above for $F_{1}$.

r) $s \notin b\left(\mathcal{C}_{1}\right) \cup b\left(\mathcal{C}_{2}\right)$. In this case no conclusion about $E_{1}$ or $F_{1}$ can be obtained with the available data.

Which of these alternatives (p), (q) and (r) is actually realized with our initial data $\left(s \in b_{1}\left(h_{0}\right)\right)$ is a question which cannot be predicted without further data. However, no contradiction is implied.

\section{References}

[1] R.B. Griffiths, J. Stat. Phys. 36, 219 (1984).

[2] R.B. Griffiths and R. Omnès, Physics Today (August 1999) 26.

[3] Quantum theory of measurement, J.A. Wheeler and W.H. Zurek (eds.) (Princeton University Press, Princeton, 1983).

[4] G. Nisticò, Found. Phys. 29, 221 (1999).

[5] A. Kent, Phys. Rev. Letters 78, 2874 (1997).

[6] R.B. Griffiths and J.B. Hartle, Phys. Rev. Lett. 81, 1981 (1998).

[7] A. Kent, Phys. Rev. Lett. 81, 1982 (1998).

[8] R.B. Griffiths, Phys. Rev. A 57, 1604 (1998).

[9] R.B. Griffiths, Consistent Quantum Theory (Cambridge University Press, Cambridge, U.K., 2002). 